\title{
Colonisation d'un récif frangeant de l'île de La Réunion par les larves de poissons coralliens
}

\section{Colonization of a fringing reef of Reunion Island by coral fish larvae}

\author{
Patrick Durville ${ }^{\mathrm{a}, \mathrm{b}, *}$, Pierre Bosc $^{\mathrm{c}}$, René Galzin ${ }^{\mathrm{b}}$, Chantal Conand ${ }^{\mathrm{a}}$ \\ ${ }^{a}$ Université de La Réunion, Laboratoire d'Ecologie Marine, BP 7151, 15 avenue René Cassin, 97715 Saint-Denis Messag. 9, La Réunion \\ ${ }^{\mathrm{b}}$ Ecole Pratique des Hautes Etudes, ESA 8046 CNRS, Université de Perpignan, 66860 Perpignan cedex, France \\ ‘Association Réunionnaise pour le Développement de l'Aquaculture, ZI Les Sables, 97427 Étang-Salé, La Réunion
}

Reçu le 25 mai 2001; reçu en forme révisée le 18 octobre 2001; accepté le 22 octobre 2001

\section{Résumé}

La colonisation d'un récif frangeant de l'île de La Réunion par les larves de poissons a lieu essentiellement entre les mois d'août et de février, avec deux maxima, en août-septembre, quand les eaux sont les plus froides et, en février lorsque la température de l'eau de mer est maximale. Le nombre d'individus ainsi que le nombre d'espèces échantillonnés présentent des valeurs similaires au cours de deux années consécutives avec néanmoins une forte variation des espèces impliquées. À La Réunion, le nombre de post-larves qui colonisent les platiers serait cent à quatre cents fois moins élevé que dans d'autres récifs de grandes dimensions mieux structurés. Ce phénomène pourrait s'expliquer par les caractéristiques géographiques de l'île. La régulation du stock de poissons adultes est due en grande partie à l'arrivée des post-larves sur les récifs et correspondrait au modèle de « recrutement limitant». (C) 2002 Ifremer/CNRS/IRD/Éditions scientifiques et médicales Elsevier SAS. Tous droits réservés.

\begin{abstract}
The colonization of a fringing reef of Reunion Island by fish larvae takes place essentially from August to February with two peaks, in August-September, when the water temperatures are coldest, and in February when they are at a maximum. The number of fishes, as well as the number of sampled species, present similar values over two consecutive years with, nevertheless, a strong variation in the species involved. In Reunion Island, the number of post-larvae which colonise the reef is one hundred to four hundred times lower than on larger and more structured reefs. This phenomenon could be explained by the geographical characteristics of the island. The stock control of the adult fish in Reunion could largely be the result of the arrival of post-larvae on reefs and would correspond to the model of "recruitment limitation”. () 2002 Ifremer/CNRS/IRD/Éditions scientifiques et médicales Elsevier SAS. All rights reserved.
\end{abstract}

Mots clés: Colonisation larvaire; Recrutement; Poisson corallien; Récif frangeant; île de La Réunion

Keywords: Larval colonization; Recruitment; Coral fish; Fringing reef; Reunion Island

\section{Introduction}

De nombreuses espèces de poissons composent la faune des récifs coralliens et participent à leur équilibre. L'étude des mécanismes responsables du maintien de cette diversité

\footnotetext{
* Auteur correspondant.

E-mail address: patrick.durville@univ-reunion.fr (P. Durville).
}

est donc essentielle. Elle concerne avant tout le cycle biologique des espèces et leurs relations avec le milieu. Les poissons récifaux généralement très féconds Sale_ 1980 possèdent pour la plupart un développement avec deux phases distinctes : un stade larvaire pélagique et un stade adulte benthique en relation avec le récif. Le renouvellement des populations locales vient donc du recrutement des larves planctoniques et de la capacité d'accueil du milieu Doherty.1982]. Depuis une dizaine d'années, grâce à la 
mise au point de la technique des "filets de crête », la phase de colonisation des milieux lagonaires par les post-larves de poissons a pu être étudiée en Polynésie Dufour et Galzin. 1992] et en Australie (Mc Ilwain. 1997) Ces résultats tendent à montrer que la colonisation est un processus actif concernant essentiellement les larves âgées ou "postlarves » capables de se métamorphoser. Le nombre de poissons colonisant les récifs est beaucoup plus important que ne laissaient paraître les études sur les juvéniles déjà recrutés et la prédation serait l'un des facteurs essentiels limitant rapidement le nombre de nouvelles recrues après leur arrivée dans le lagon HHixon. 1991. La variabilité spatio-temporelle de la colonisation des récifs, en partie responsable de la variabilité spatio-temporelle de la structure des populations adultes (Doherty et Fowler, 1994a), est actuellement expliquée par deux modèles de régulation des populations :

- un modèle de «recrutement limitant» basé sur la limitation de l'apport larvaire sur les récifs alors que les ressources et l'habitat sont toujours en quantité suffisante Doherty 1982: Victor 1986: Doherty et Fowler_1994a

- un modèle de «compétition » basé sur la compétition interspécifique Sale 1980. Sale_et Dowolas 1984 et intraspécifique CAnderson et al. 1981: Shulman et al. 1984 pour les ressources et l'habitat, avec une présence de post-larves en excès sur les récifs.

Les populations adultes seraient en fait régulées par le modèle de « recrutement limitant » jusqu'à un certain seuil. Lorsque ce seuil serait atteint ou dépassé, le modèle de «compétition» interviendrait Caselle, 1999; Shima 1999.

La Réunion, mesurant près de $70 \mathrm{~km}$ selon son axe NO-SE et environ $50 \mathrm{~km}$ suivant l'axe NE-SO, est une île jeune du point de vue géologique (3 à 5 millions d'années). Située au Sud-Ouest de l'océan Indien par $21^{\circ} 07^{\prime}$ de latitude Sud et $55^{\circ} 32^{\prime}$ de longitude Est, à environ $300 \mathrm{~km}$ au Nord du tropique du Capricorne et à plus de $800 \mathrm{~km}$ à l'Est de Madagascar, elle constitue, avec les îles Maurice et Rodrigues, l'archipel des Mascareignes (Fig. 1). Les formations récifales de La Réunion sont de type frangeant, avec une largeur maximale de $500 \mathrm{~m}$ et une profondeur de $1,50 \mathrm{~m}$. Elles sont présentes sur le littoral occidental et constituent une ceinture discontinue de près de $25 \mathrm{~km}$, pour une surface de $12 \mathrm{~km}^{2}$. Etudiés depuis plus de vingt ans, ces platiers récifaux présentent l'avantage de former un terrain d'expérimentation de taille réduite facile à appréhender, notamment pour l'étude des espèces vagiles comme les poissons. Letourneur (1992) supposait déjà que le recrutement conditionnait une grande partie des fluctuations temporelles observées sur les peuplements ichtyologiques des récifs de l'île, alors que parallèlement, Chabanet et al 1996) montraient que la destruction de l'habitat perturbait le recrutement en juvéniles. Cette étude a donc été entreprise afin de quantifier l'arrivée des post-larves sur un des platiers de l'île et d'essayer de déterminer le mode de

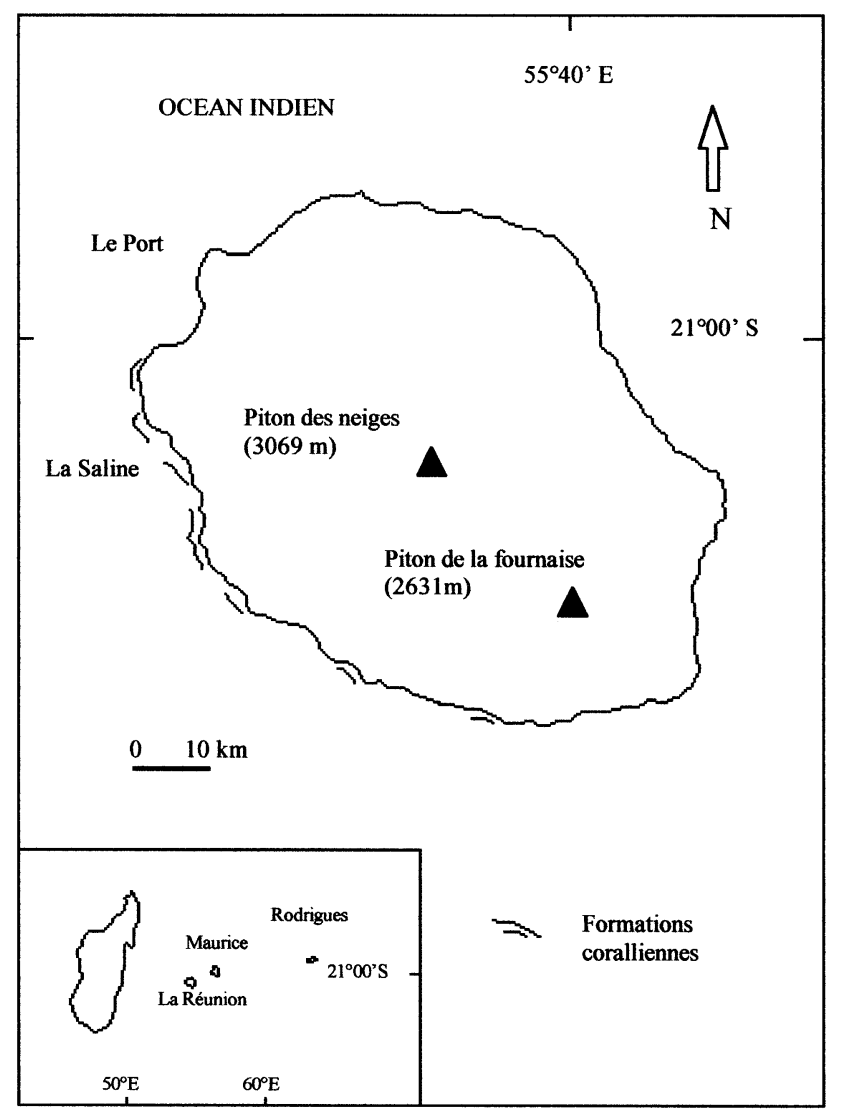

Fig. 1. Situation géographique de l'île de La Réunion et emplacement du site d'étude : le récif de la Saline.

régulation principal des populations adultes de poissons coralliens à La Réunion.

\section{Matériel et méthodes}

L'échantillonnage a été effectué sur le récif de la Saline situé sur la côte ouest de l'île. C'est le secteur corallien le plus développé et le mieux structuré de La Réunion, avec une largeur d'environ $500 \mathrm{~m}$ dont 100 à $150 \mathrm{~m}$ de platier externe. C'est également le récif où le recrutement serait le plus important Letournewr, 1996. L'échantillonnage des post-larves a été effectué au moyen d'un filet de crête de $2 \mathrm{~m}$ $\times 1 \mathrm{~m}$ d'ouverture rectangulaire pour une longueur totale de $5 \mathrm{~m}$ et une maille de $2 \mathrm{~mm}$ de coté (Fig. 2). Ce filet est prolongé d'un collecteur conique en toile, d'une longueur de $3 \mathrm{~m}$ avec une maille de $1 \mathrm{~mm}$ de côté, où sont piégés les poissons. L'ensemble est fixé perpendiculairement au déferlement par des pieux métalliques et un système d'élingues. Ce filet fonctionne comme un échantillonneur passif en filtrant en continu le flux d'eau entrant sur le platier. Le faible marnage sur l'île de La Réunion modifie peu le niveau de l'eau et seule la houle est susceptible d'induire des variations importantes du flux. 


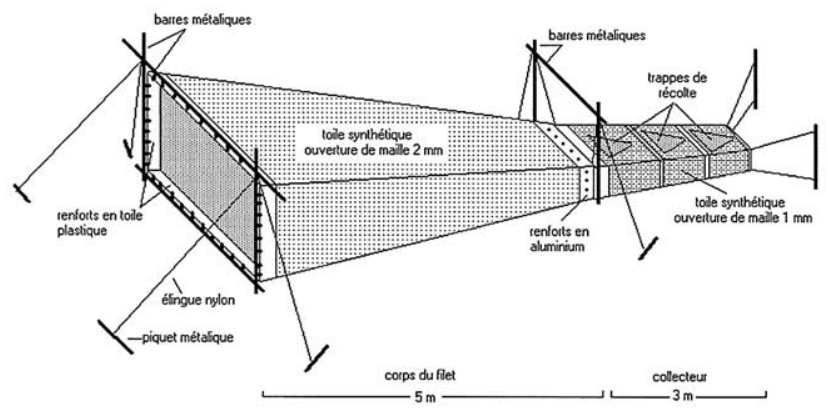

Fig. 2. Filet de crête utilisé sur le récif de la Saline.

Les prélèvements ont été effectués durant dix huit cycles lunaires de décembre 1997 à mai 1999. La plupart des auteurs qui ont étudié les variations temporelles de la colonisation larvaire ichtyologique ont montré que les périodes les plus favorables à la migration des poissons étaient les nuits de nouvelle lune Victor. 1986: Dufour ef Galzin.1992]. Le filet a donc été installé à chaque cycle lunaire, durant les cinq nuits encadrant la nouvelle lune. Tous les éléments piégés dans le collecteur ont été récoltés chaque matin et placés dans un container d'eau de mer. Le tri des post-larves de poissons a ensuite été effectué en laboratoire. Les poissons morts ont été conservés dans une solution de formol à $5 \%$ et mis en collection, alors que les post-larves vivantes ont été placées en bassin de grossissement. Ce procédé offre l'avantage de suivre l'évolution des poissons après leur métamorphose complète, jusqu'à un stade juvénile où la plupart deviennent facilement identifiables. L'identification est un des problèmes clés de l'étude des post-larves. Elle a pu être menée ici à la fois sur des critères morphologiques (taille, forme, couleur) et sur des critères comportementaux (nage, déplacements), caractéristiques des différentes espèces de poissons coralliens. Après plusieurs vérifications, cette méthode a permis de déterminer avec précision la plupart des individus capturés. Lorsqu' un doute subsistait, la détermination a été réalisée au niveau du genre ou de la famille.

Pour évaluer la représentativité de la station de prélèvement et vérifier l'homogénéité de l'arrivée des post-larves sur l'ensemble du platier de la Saline, une campagne de pré-échantillonnage à l'aide d'engins plus petits a été réalisée. Quatre petits filets identiques en tissu nylon avec une maille de $1 \mathrm{~mm}$ de coté, d'ouverture rectangulaire de $1 \mathrm{~m} \times 0,5 \mathrm{~m}$ et de longueur totale de $3 \mathrm{~m}$, ont été installés parallèlement au front récifal séparés par un intervalle de $100 \mathrm{~m}$. Un total de 190 post-larves a été capturé en dix jours d'échantillonnage. Un test de comparaison des moyennes (Krusskal-Wallis) a permis de montrer qu'il n'y avait pas de différence significative entre les quatre filets $(\mathrm{Q}=0,66$; $\mathrm{ddl}=3,39 ; p<0,05)$ et que l'arrivée des post-larves sur ce secteur du récif pouvait être considérée comme homogène.

\section{Résultats}

\subsection{Abondance de la colonisation larvaire ichtyologique}

Sur l'ensemble des échantillons collectés sur le platier récifal de la Saline durant 18 mois consécutifs, 984 postlarves ont été obtenues en 90 jours de prélèvement, avec un maximum de 54 post-larves par jour et un minimum de zéro. Près de $90 \%$ des échantillons journaliers contenaient de 0 à 20 post-larves.

L'étude de l'abondance de la colonisation mensuelle montre une arrivée importante des post-larves du mois d'août au mois de février (Fig. 3), et une colonisation plus faible entre mars et juillet. En 1998, seulement 10,8 \% des captures ont été réalisées au cours de cette dernière période. Un test U de Mann-Whitney de comparaison de moyennes montre que la différence de colonisation entre les mois d'août à février et les mois de mars à juillet est hautement significative $(\mathrm{U}=1936 ; \mathrm{ddl}=1,89 ; p<0,001)$. La moyenne journalière du nombre de post-larves pendant les mois de forte colonisation est de $17 \pm 10,4$ indiv $\mathrm{d}^{-1}$, alors qu'elle n'est que de $3,3 \pm 2,7$ indiv $\mathrm{d}^{-1}$ pendant les mois de faible colonisation, soit 5,1 fois inférieure. Il existe également deux pics bien distincts d'arrivée des post-larves : en août et en février, avec un maximum de 127 post-larves pour les cinq jours d'échantillonnages effectués au mois de février 1999, soit une moyenne de 25,4 $\pm 11,9$ indiv d $\mathrm{d}^{-1}$ durant cette période.

Les courbes d'abondance établies en 1998 et en 1999 sur six mois d'échantillonnage (décembre à mai) indiquent une évolution similaire du nombre de post-larves arrivant sur les platiers au cours des deux années (Fig. 4a). Un test de corrélation de Spearman, qui permet de mesurer le degré d'association entre deux variables, montre une corrélation significative entre les deux courbes, avec un coefficient Rs $=0,73(p<0,001)$.

\subsection{Richesse spécifique de la colonisation larvaire ichtyologique}

Les post-larves échantillonnées appartiennent à 39 familles et 98 espèces (Table 1). Les Gobiidae sont les plus

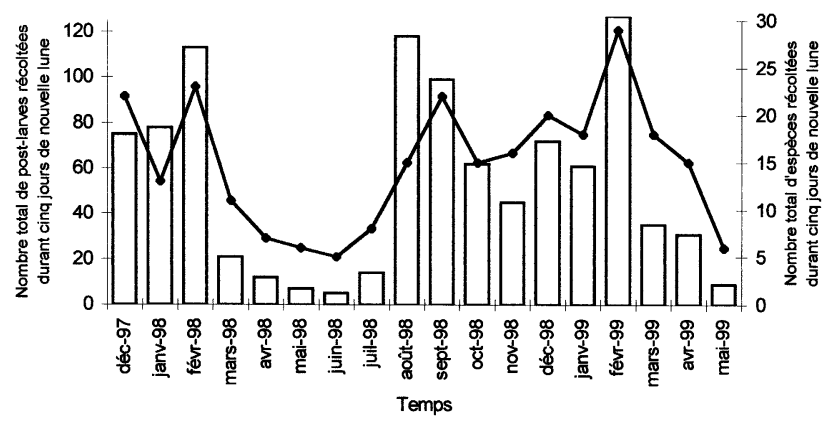

Fig. 3. Variation de l'abondance (histogramme) et de la richesse spécifique (courbe) de la colonisation par les post-larves de poissons en fonction du temps. 

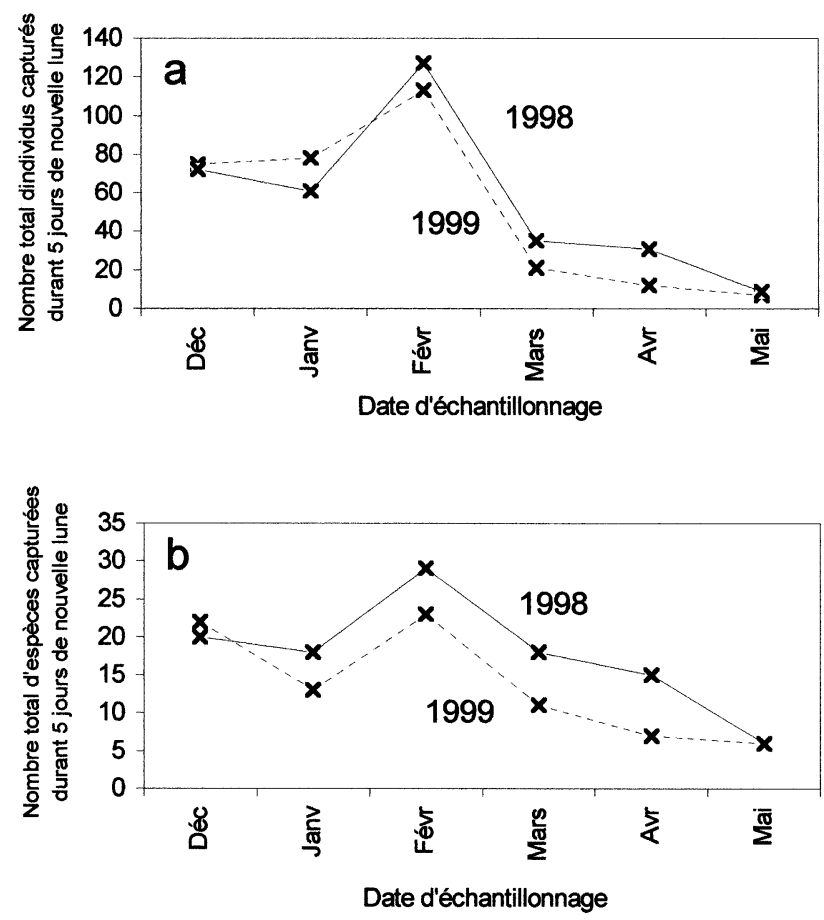

Fig. 4. Comparaison du nombre d'individus (a) et du nombre d'espèces (b) de post-larves de poissons capturées en 1998 et 1999 durant 6 périodes d'échantillonnages.

abondants avec $22 \%$ des captures totales, viennent ensuite les Pomacentridae $(17 \%)$ et les Acanthuridae $(11 \%)$. Les Apogonidae, les Scaridae, les Labridae, les Blenniidae, les Holocentridae, les Mullidae et les Scorpaenidae totalisent plus de $33 \%$ du nombre d'individus. L'ensemble des 19 autres familles ne représente que $15 \%$ des captures. La majorité des espèces $(96 \%)$ colonisent les platiers du mois d'août au mois de février avec deux pics observés : au mois de septembre et au mois de février (Fig. 3). Un test U de Mann-Whitney montre que la différence de colonisation larvaire entre les mois d'août à février et les mois de mars à juillet est hautement significative $(\mathrm{U}=1873$; ddl $=1,89$; $p<0,001)$. La moyenne journalière du nombre d'espèces pendant les mois de forte colonisation est de $7,3 \pm 2,7 \mathrm{sp} \mathrm{d}^{-1}$, alors qu'elle n'est que de 2,6 $\pm 1,8 \mathrm{sp} \mathrm{d}^{-1}$ pendant les mois de faible colonisation, soit 2,8 fois inférieure.

L'évolution temporelle de la richesse spécifique réalisée sur 6 mois d'échantillonnage (décembre à mai) montre la même tendance en 1998 et en 1999, avec une corrélation hautement significative des deux courbes, Rs $=0,76$ $(p<0,001)$ (Fig. 4b). Il existerait donc chaque année des périodes données où de nombreuses espèces coloniseraient les platiers. Cependant, le coefficient de contingence de Jaccard, qui permet de comparer de façon qualitative la colonisation sur ces deux années, montre que ce ne sont pas forcément les mêmes espèces qui sont observées d'une année sur l'autre $(\mathrm{Cj}=0,46)$.
Table 1

Nombre d'espèces, nombre d'individus et pourcentage de post-larves de poissons échantillonnées par famille, classées par ordre décroissant

\begin{tabular}{|c|c|c|c|}
\hline Familles & $\begin{array}{l}\text { Nombre } \\
\text { d'espèces }\end{array}$ & $\begin{array}{l}\text { Nombre } \\
\text { d'individus }\end{array}$ & $\begin{array}{l}\% \text { d'individus par } \\
\text { rapport au total }\end{array}$ \\
\hline Gobiidae & 6 & 219 & 22,26 \\
\hline Pomacentridae & 12 & 170 & 17,28 \\
\hline Acanthuridae & 10 & 112 & 11,38 \\
\hline Apogonidae & 6 & 60 & 6,10 \\
\hline Scaridae & 1 & 59 & 6,00 \\
\hline Labridae & 6 & 57 & 5,79 \\
\hline Blenniidae & 6 & 51 & 5,18 \\
\hline Holocentridae & 3 & 39 & 3,96 \\
\hline Mullidae & 3 & 31 & 3,15 \\
\hline Scorpaenidae & 5 & 29 & 2,95 \\
\hline Chaetodontidae & 5 & 20 & 2,03 \\
\hline Bothidae & 1 & 20 & 2,03 \\
\hline Balistidae & 2 & 17 & 1,73 \\
\hline Muraenidae & 2 & 13 & 1,32 \\
\hline Synodontidae & 2 & 10 & 1,02 \\
\hline Antennariidae & 3 & 8 & 0,81 \\
\hline Monodactylidae & 1 & 7 & 0,71 \\
\hline Ophichthidae & 1 & 5 & 0,51 \\
\hline Engraulidae & 1 & 5 & 0,51 \\
\hline Plesiopidae & 1 & 5 & 0,51 \\
\hline Mugilidae & 1 & 5 & 0,51 \\
\hline Syngnathidae & 3 & 4 & 0,41 \\
\hline Gerreidae & 1 & 4 & 0,41 \\
\hline Belonidae & 1 & 3 & 0,30 \\
\hline Kuhliidae & 1 & 3 & 0,30 \\
\hline Ostraciidae & 1 & 3 & 0,30 \\
\hline Anguillidae & 1 & 2 & 0,20 \\
\hline Gobiesocidae & 1 & 2 & 0,20 \\
\hline Aulostomidae & 1 & 2 & 0,20 \\
\hline Fistulariidae & 1 & 2 & 0,20 \\
\hline Microdesmidae & 1 & 2 & 0,20 \\
\hline Zanclidae & 1 & 2 & 0,20 \\
\hline Dactylopteridae & 1 & 1 & 0,10 \\
\hline Lethrinidae & 1 & 1 & 0,10 \\
\hline Polynemidae & 1 & 1 & 0,10 \\
\hline Siganidae & 1 & 1 & 0,10 \\
\hline Soleidae & 1 & 1 & 0,10 \\
\hline Monacanthidae & 1 & 1 & 0,10 \\
\hline Tetraodontidae & 1 & 1 & 0,10 \\
\hline indeterminés & - & 6 & 0,61 \\
\hline Total (39) & 98 & 984 & $100 \%$ \\
\hline
\end{tabular}

\subsection{Similitudes entre les évolutions des données qualitatives et quantitatives}

La richesse spécifique et l'abondance post-larvaire varient de façon similaire en fonction du temps. Les pics de richesse spécifique correspondent presque toujours aux pics d'abondance, notamment au mois de février. Un test de corrélation de Spearman entre les données qualitatives et quantitatives sur l'ensemble de la période d'échantillonnage montre que cette corrélation est hautement significative $\mathrm{Rs}=0,92(p<0,001)$. Aussi, une majorité d'espèces colonisent donc les platiers, en grand nombre, au même moment. Cependant, sur les 42 espèces présentes durant les deux pics de colonisation en février 1998 et en février 1999 , seules 11 d'entre elles, soit seulement $26 \%$, sont communes aux deux années. Toutes les espèces ne se retrouvent donc pas d'une année sur l'autre. Quant au pic de richesse 
Table 2

Nombre d'échantillons, nombre total de post-larves de poissons, abondance moyenne journalière, nombre total d'espèces, moyenne journalière d'espèces échantillonnées et nombre d'espèces communes (au moins 4 échantillons sur 5) durant deux pics de colonisation aux mois de février 1998 et 1999

\begin{tabular}{lcc}
\hline & Février 1998 & Février 1999 \\
\hline $\begin{array}{l}\text { Nombre d'échantillons } \\
\text { (jours de prélèvement) }\end{array}$ & 5 & 5 \\
$\begin{array}{l}\text { Nombre total de post-larves } \\
\text { échantillonnées }\end{array}$ & 113 & 127 \\
$\begin{array}{l}\text { Abondance moyenne journalière } \\
\text { Nombre total d'espèces }\end{array}$ & $22,6 \pm 12,7$ & $25,4 \pm 11,9$ \\
échantillonnées & 24 & 29 \\
$\begin{array}{l}\text { Moyenne journalière d'espèces } \\
\text { échantillonnées } \\
\text { Nombre d'espèces communes } \\
\text { (au moins 4 échantillons sur 5) }\end{array}$ & $9 \pm 2,9$ & $10,2 \pm 4,6$ \\
\hline
\end{tabular}

spécifique du mois de septembre 1998, seules 6 espèces sont communes avec l'ensemble des 42 espèces des mois de février 1998 et février 1999. Des espèces différentes arrivent ainsi sur les platiers au début et à la fin de la période de colonisation.

Une analyse détaillée de la colonisation larvaire durant les deux pics de février 1998 et février 1999 montre que les valeurs d'abondance et de richesse spécifique observées chaque jour sont très dispersées par rapport à la moyenne (Table 2). Seules 3 espèces en février 1998 (Naso unicornis, Zebrasoma desjardinii, Sargocentron diadema) et 2 en février 1999 (Naso unicornis, Sargocentron diadema) sont observées pendant au moins 4 jours d'échantillonnage sur 5 . Il existerait donc des périodes de colonisation bien définies pour ces espèces, tandis que la majorité des autres espèces, coloniseraient les platiers à des périodes variables.

\subsection{Modalité de la colonisation larvaire des principales familles}

La Fig. 5montre le nombre d'individus capturés, pour les 10 familles les plus abondantes (soit $83 \%$ de l'effectif total), en fonction du temps. La colonisation est principalement centrée sur les mois d'août à février, avec des pics d'abondance très marqués chez les Gobiidae et les Acanthuridae, alors que d'autres familles, comme les Apogonidae, présentent une colonisation moins massive mais plus régulière dans le temps. Les Gobiidae et les Pomacentridae sont présents une grande partie de l'année, alors que les Acanthuridae, les Scaridae ou les Holocentridae ont des périodes de colonisation plus limitées. La plupart des familles ayant des post-larves de petites tailles au moment de la colonisation, comme les Gobiidae, les Scaridae, les Labridae ou les Blenniidae, ont un pic de colonisation en août, septembre ou octobre alors que celles ayant des post-larves de grandes tailles, comme les Acanthuridae ou les Holocentridae, colonisent les platiers plus tardivement, en février-mars.
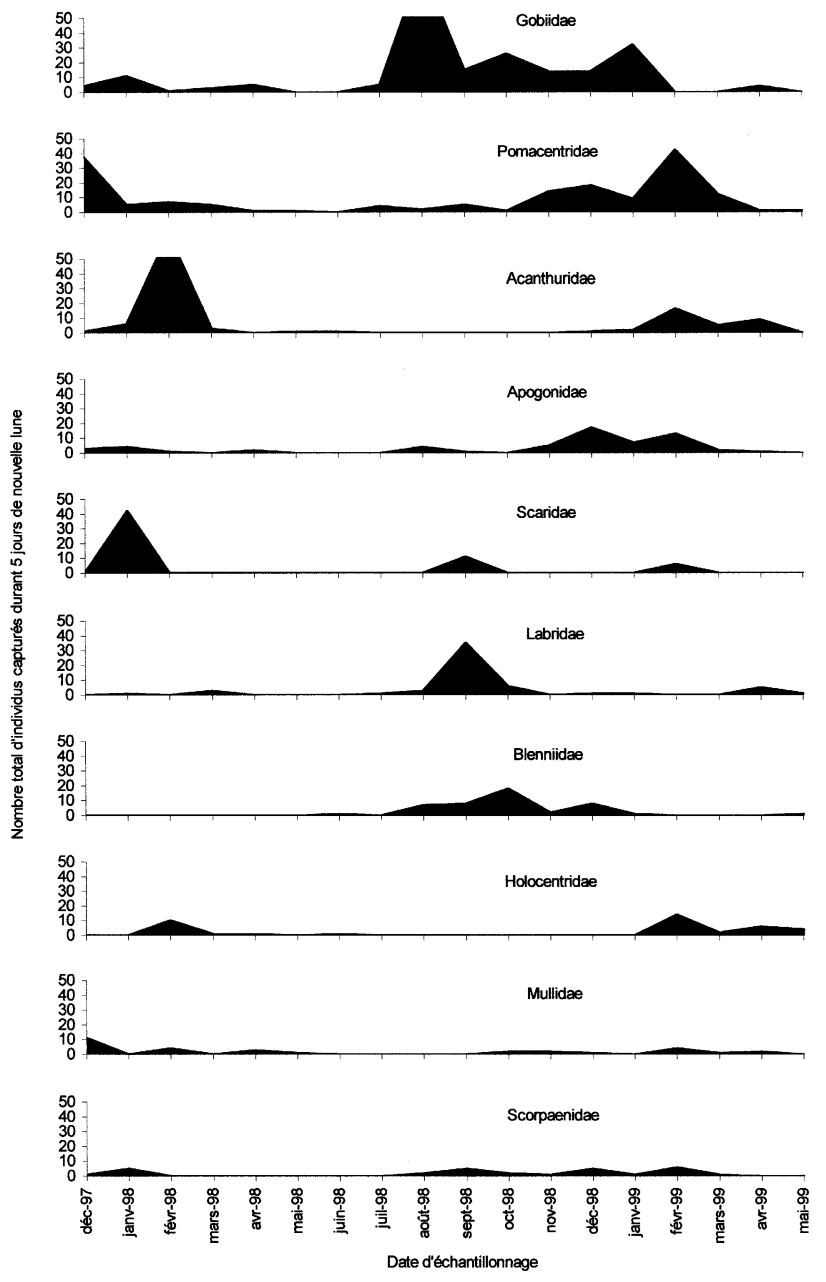

Fig. 5. Nombre d'individus capturés au cours du temps pour les 10 familles de poissons les plus abondantes. Le classement est décroissant en fonction du nombre total d'individus capturés.

\section{Discussion}

\subsection{Richesse et abondance de la colonisation par les post-larves de poissons}

Plus de $95 \%$ des individus échantillonnés sur le récif de la Saline appartiennent à des espèces caractéristiques des zones de platier de La Réunion inventoriées par Letourneu 1992) et Chahanet (1994). Seules quelques familles rencontrées régulièrement dans ces milieux n'ont jamais été présentes dans nos prélèvements comme les Carangidae ou les Serranidae. Ces dernières recrutent peut-être dans un autre milieu ou ont échappé à l'échantillonnage, soit à cause de leur trop faible abondance, soit en adoptant des comportements particuliers (grégarisme, évitement du filet), soit en empruntant d'autres passages (contre-courants au niveau des passes) ou enfin en ayant des périodes de colonisation limitées dans le temps. Chabanet (1994) détaille le peuplement de poissons adultes observé visuellement sur le platier de la Saline et montre que les familles les mieux représentées sont les Acanthuridae (34,4\%), les Scaridae (27,6\%), 
Table 3

Comparaison du nombre moyen de post-larves capturées par jour sur différents sites coralliens, à la même saison, en utilisant la technique des filets de crête

\begin{tabular}{|c|c|c|c|c|}
\hline Lieu & Auteur et date de l'étude & Ouverture du filet & Période d'échantillonnage & $\begin{array}{l}\text { Nombre d'individus par jour ramené } \\
\text { au mètre linéaire de crête récifale }\end{array}$ \\
\hline $\begin{array}{l}\text { Rangiroa } \\
\text { Polynésie }\end{array}$ & Dufour 1992 & $1 \times 0,25 \mathrm{~m}$ & février/mars 1989 & 2117 \\
\hline $\begin{array}{l}\text { Lizard Island } \\
\text { Eastern Australia }\end{array}$ & Dufour 1992 & $1 \times 0,25 \mathrm{~m}$ & février/mars 1991 & 3996 \\
\hline $\begin{array}{l}\text { Ningaloo reef } \\
\text { Western Australia }\end{array}$ & McIlwain 1997 & $1 \times 1,5 \mathrm{~m}$ & décembre/janvier 1995/1996 & 1083 \\
\hline Réunion & $\begin{array}{l}\text { Durville présente étude } \\
\text { Durville présente étude }\end{array}$ & $\begin{array}{l}2 \times 1 \mathrm{~m} \\
2 \times 1 \mathrm{~m}\end{array}$ & $\begin{array}{l}\text { janvier/février } 1998 \\
\text { janvier/février } 1999\end{array}$ & $\begin{array}{l}9,5 \\
9,4\end{array}$ \\
\hline
\end{tabular}

les Pomacentridae $(24,4 \%)$, les Chaetodontidae $(7,8 \%)$ et les Mullidae $(2,2 \%)$. Ces trois premières familles sont également parmi les plus abondantes des post-larves échantillonnées dans cette étude. La technique du filet de crête pourrait alors donner une bonne image d'une partie des peuplements en place et servir de complément à d'autres méthodes d'échantillonnage, notamment pour les espèces de petite taille (Gobiidae) ou les espèces nocturnes (Apogonidae) qui sont rarement échantillonnées par observations visuelles alors qu'elles sont capturées par le filet de crête.

L'abondance des post-larves de poissons obtenue avec le filet de crête sur le platier de la Saline semble très différente de celles obtenues avec la même technique sur d'autres types de récifs coralliens (Table 3). Il est difficile de comparer un nombre d'individus capturés avec des filets différents, mais la colonisation à La Réunion est nettement plus faible que sur les autres récifs. Ramené au nombre d'individus échantillonnés sur un mètre linéaire de crête récifale, la colonisation post-larvaire sur le platier de la Saline, qui se ferait pourtant de façon homogène, serait 100 à 400 fois moins importante que sur certains récifs du Pacifique Dufour. 1992: Mc Jlwain. 1997. Les conditions hydrodynamiques extrêmement variables sur les platiers de l'île ont peut-être influencé l'échantillonnage, mais sur l'ensemble des prélèvements effectués aucun signe de colonisation significativement plus importante n'est apparu. Cette très faible colonisation peut provenir de la situation géographique de l'île. Relativement isolée $(230 \mathrm{~km}$ de Maurice, $732 \mathrm{~km}$ de Madagascar et $830 \mathrm{~km}$ de Rodrigues), La Réunion est sous l'influence du courant sud-équatorial s'écoulant à une vitesse moyenne de 0,5 nœuds d'Est en Ouest sur près de $2000 \mathrm{~km}$ de large. Ce courant qui traverse peu de formations coralliennes depuis l'Australie, située à plus de $5000 \mathrm{~km}$, est probablement pauvre en larves de poissons récifaux lorsqu'il arrive aux alentours de l'île. De plus, comme le montrent Letourneur et al_(1998) le recrutement est fortement corrélé au transport des larves dans les eaux de surface qui dépend des vents dominants. À La Réunion, le régime des vents est surtout représenté par les Alizés qui soufflent d'un secteur Sud-Est en saison fraîche et évoluent vers l'Est en saison chaude. Ceci entraine une circulation générale de surface venant du Sud - Sud-Est tout au long de l'année, drainant des eaux océaniques pauvres en plancton Jacques et Treguer.1986.
De ce fait, on peut supposer qu'une grande partie des poissons colonisant les platiers de l'île proviendrait des populations locales, les larves demeurant à proximité du récif grâce à l'existence de tourbillons océaniques dû à un « effet d'île » pouvant jouer le rôle d'attracteurs SWearer ed al. 1999). Planes et al. (1996) ont montré que ce phénomène d' " auto-recrutement » était courant dans les îles de Polynésie pour certaines espèces comme Acanthurus triostegus. Il est donc probable, selon cette hypothèse, que la taille des récifs et leur isolement soient déterminants pour le recrutement des populations de poissons. En effet, à La Réunion, les récifs ne représentent qu'une superficie de $12 \mathrm{~km}^{2}$ pour l'ensemble de l'île. Le succès de la reproduction des peuplements ichtyologiques, soumis par ailleurs à un effort de pêche intensif dans ces milieux restreints, ne peut être équivalent à celui de formations coralliennes plus développées. La quantité de nouvelles recrues colonisant les récifs pourrait être limitée et devenir inférieure à la disponibilité des ressources alimentaires et à la capacité d'accueil du milieu. C'est donc le modèle de « recrutement limitant » qui expliquerait l'abondance des différentes espèces constituant les populations de poissons adultes des platiers de $\mathrm{La}$ Réunion.

\subsection{Variabilité temporelle de la colonisation par les post- larves de poissons}

Le caractère saisonnier de la colonisation larvaire, avec une arrivée massive des post-larves de poissons coralliens en saison chaude, a déjà été décrit, aussi bien dans l'hémisphère Nord que dans l'hémisphère Sud Russel et al, 1977. Williams 1983 · Mc Farland et Ogden, 1985). La courbe des températures moyennes mensuelles de l'eau de mer à La Réunion (Conand, com. pers.) permet de mieux définir les périodes d'arrivée des post-larves (Fig. 6). Le maximum de colonisation a lieu lorsque la température de l'eau de mer augmente : du mois d'août au mois de février, avec deux maxima correspondant à l'arrivée de différentes espèces, en août-septembre quand les eaux sont les plus froides et en février lorsque la température est maximale. Dès que celle-ci diminue, la colonisation baisse brutalement alors que la température est encore élevée $\left(27,5^{\circ} \mathrm{C}\right.$ au mois de mars). C'est donc plus l'inversion de la température plutôt que sa valeur qui déterminerait ce phénomène. Il existe, de 


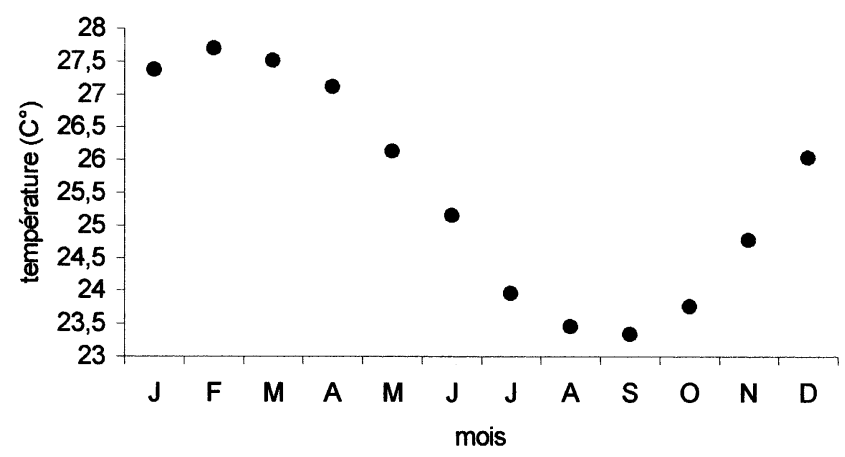

Fig. 6. Moyennes mensuelles de la température de sub-surface de l'eau de mer, établies au Port de 1993 à 2000, à partir de la moyenne des relevés horaires (Conand F., comm. pers.).

façon générale sur les récifs, une diminution de l'arrivée des post-larves lorsque la température de l'eau diminue mais la colonisation reste présente tout au long de l'année. Elle est en moyenne 5,1 fois moins importante durant les mois de mars à juillet sur le platier de la Saline à La Réunion. Williams (1983) constate sur la Grande Barrière d'Australie que le nombre de poissons recrutés peut varier d'un facteur 6 entre les pics d'une même année, alors que Dufour ed Galzin (1992) n'observent qu'une colonisation deux fois moins importante pendant l'hiver austral en Polynésie. L'amplitude de variation entre les saisons semble donc différente selon les régions. Williams (1983) montre que dans les îles de la Grande Barrière d'Australie, cette variabilité est importante, alors que dans les Caraïbes tropicales elle est moins marquée Luckhurst et Luckhurst. 1977: Mc Farland et Ogden. 1985) Il est probable que le caractère saisonnier de la colonisation soit d'autant plus prononcé que les saisons sont plus distinctes, comme c'est le cas à La Réunion. Ce phénomène pourrait alors être lié à des périodes de pontes peu étalées dans le temps ou irrégulières, ce qui, d'après Lasker (1989) n'est pourtant pas le cas pour la majorité des poissons récifaux, où plusieurs pontes peuvent parfois se succéder au cours d'une même saison. À La Réunion, peu d'études ont été réalisées sur la reproduction des poissons coralliens, à l'exception de Letournewr (1992) qui évalue la période de reproduction de Stegastes nigricans vers les mois de mai-juin et octobre, ce qui pourrait correspondre au retour des post-larves de cette espèce qui ont été échantillonnées en grand nombre en novembre et en février.

Plusieurs études ont mis en évidence de fortes variations inter-annuelles dans le recrutement des poissons récifaux Williams, 1983 : Eckert 1984. Sale et al, 1984: Walsh 1987) À La Réunion, Letournewr (1992) émet l'hypothèse d'un recrutement plus ou moins important pour expliquer les variations d'abondance et de diversité qu'il observe sur les platiers d'une année sur l'autre. Dans notre étude, la colonisation larvaire est sensiblement équivalente, autant en abondance qu'en richesse spécifique, en 1998 et 1999, avec cependant une forte variation spécifique. Dufour (1997) estime que le nombre de post-larves colonisant un récif donné, une année donnée, se fait au hasard de leur survie dans l'océan et que cela ne permet donc pas de prédire quel sera ce nombre dans une autre île ou l'année suivante. Si ceci est vrai en ce qui concerne l'abondance des post-larves, la période de colonisation de certaines espèces semble cependant être prévisible, comme Naso unicornis ou Sargocentron diadema, qui coloniseraient les platiers de La Réunion chaque année aux mêmes périodes. Williams 1983 retrouve également des pics de recrutement de Pomacentridae au même moment d'une année sur l'autre. Les fluctuations éventuelles du recrutement seraient alors essentiellement dûes aux facteurs environnementaux comme le passage d'un cyclone par exemple Bouchon et al.. 1991: Letourneur et al.. 1998) ou l'état de dégradation du milieu corallien CChabanet et al. 1996)

\section{Conclusion}

La compréhension des procédés impliqués dans la structuration des peuplements adultes de poissons récifaux a considérablement évoluée ces dernières années. La compétition avait longtemps été considérée comme le facteur déterminant l'abondance et la diversité ichtyologique dans un milieu récifal (modèle de « compétition » Sale, 1980. Shulman et_al, 1984), mais les travaux actuels tendent à montrer que si les phénomènes de compétition ont effectivement un impact, il est variable selon l'importance du recrutement (modèle de « recrutement limitant » Doherty 1982). Les résultats obtenus sur un récif frangeant de La Réunion montrent que le fonctionnement des peuplements de poissons serait de type « recrutement limitant». L'approvisionnement en post-larves étant réduit, la compétition intra et interspécifique serait alors théoriquement limitée pour les nouvelles recrues et la capacité d'accueil du milieu ainsi que les ressources alimentaires pourraient être considérées comme excédentaires par rapport à l'apport de post-larves. C'est en fait la taille relative du système récifal considéré qui doit être prise en compte. Dufour (1992 trouve sur le site de Tiahura à Moorea une densité de nouvelles recrues de 706,3 ind $\mathrm{m}^{-2} \mathrm{an}^{-1}$ en considérant une répartition des post-larves sur toute la surface du lagon. À La Réunion, cette densité répartie sur la surface du platier de la Saline s'élèverait seulement à 4,83 ind $\mathrm{m}^{-2} \mathrm{an}^{-1}$ pour l'année 1998, ce qui semblerait confirmer la disponibilité des ressources et de l'habitat pour les post-larves colonisant ce milieu.

Contrairement aux récifs d'archipels plus importants comme en Polynésie où la colonisation s'effectue massivement et peut provenir des îles environnantes Dufour et al. 1996) la situation géographique relativement isolée de La Réunion et l'hydrodynamisme général autour de l'île, sont probablement responsables d'une faible colonisation allochtone. Seuls des phénomènes météorologiques ou hydrody namiques particuliers seraient à l'origine d'arrivées massives inhabituelles de post-larves, comme cela s'est produit en avril 1994 pour Epinephelus merra (Letourneur et al., 
1998). Jones et al. (1999) montrent que l'auto-recrutement est couramment observé sur les récifs coralliens et que 15 à $60 \%$ des juvéniles de certaines espèces peuvent être issus des populations locales. Dans cette configuration, le stock de poissons coralliens pour l'ensemble de l'île serait implicitement en étroite relation avec la dimension des récifs, d'où une colonisation moindre par rapport aux formations coralliennes plus importantes et/ou moins isolées. Doherty et Fowler (1994b) ont montré sur la Grande Barrière d'Australie que les récifs ayant un taux de colonisation faible, restent plus pauvres en adultes que les autres. Ce phénomène ne serait pourtant pas confirmé à La Réunion, puisque Letourneur (1992) trouve une densité moyenne de poissons sur les platiers de l'île équivalente à celle de Moorea en Polynésie qui possède un fort taux de colonisation. Néanmoins, les populations adultes de poissons récifaux de La Réunion, régulées par un apport larvaire limité et subissant probablement de façon marquée les différentes fluctuations du recrutement, pourraient être en équilibre fragile et seraient ainsi encore plus sensibles aux effets de la pêche que des récifs plus vastes ou en connexion avec d'autres.

\section{Acknowledgements}

Le financement de cette étude a été assuré par le Conseil Régional de La Réunion, le Fonds Social Européen, 1'Association Réunionnaise pour le Développement de l'Aquaculture (ARDA) et le Laboratoire d'Ecologie Marine (ECOMAR) de l'Université de La Réunion.

\section{References}

Anderson, G.R.V., Ehrlich, A.P., Ehrlich, P.R., Roughgarden, J.D., Russel, B.C., Talbot, F.H., 1981. The community structure of coral reef fishes. Am. Nat. 117, 476-495.

Bouchon, C., Bouchon-Navaro, Y., Imbert, D., Louis, M., 1991. Effets de l'ouragan Hugo sur les communautés côtières de Guadeloupe (Antilles françaises). Ann. Inst. Océanogr. 67, 5-33.

Caselle, J.E., 1999. Density-dependent early post-settlement mortality in a coral reef fish and its effect on local population size. Ecol. Monogr. 69, 177-194.

Chabanet, P., 1994. Etude des relations entre les peuplements benthiques et les peuplements ichtyologiques sur le complexe récifal de StGilles/La Saline à l'île de La Réunion. Thèse Envir. Marin, Univ. Aix-Marseille III. pp. 235.

Chabanet, P., Dufour, V., Galzin, R., 1996. Disturbance impact on reef fish communities in Reunion Island (Indian Ocean. J. Exp. Mar. Biol. Ecol. 188, 29-48.

Doherty, P.J., 1982. Some effects of density of the juveniles of two species of tropical territorial damselfishes. J. Exp. Mar. Biol. Ecol. 65, 249-261.

Doherty, P.J., Fowler, A.J., 1994. An empirical test of recruitmentlimitation in a coral reef fish. Science 263, 935-939.

Doherty, P.J., Fowler, A.J., 1994. Demographic consequences of variable recruitment: a congeneric comparison of two damselfishes. Bull. Mar. Sci. 54, 297-313.

Dufour, V., 1997. Oward informed management of Coral Reef Fish Populations. Fr. Sci. Technol. 29, 5.
Dufour, V., Galzin, R., 1992. Le recrutement des poissons récifaux de Polynésie française, impact sur la dynamique des peuplements et conséquences sur la gestion des stocks. Cybium 16 (4), 267-277.

Dufour, V., Riclet, E., Lo-Yat, A., 1996. Colonization of reef fishes at Moorea Island, French Polynesia: Temporal and spatial variation of the larval flux. Mar. Fresh. Res. 47, 413-422.

Eckert, G.J., 1984. Annual and spatial variation in recruitment of Labroid fishes among seven reefs in the Capricorn bunker group. Great Barrier reef. Mar. Biol. 78, 123-127.

Hixon, M.A., 1991. Predation as a process structuring coral reef fish communities. In : Sale, P (Ed.), The Ecology of Fishes on Coral Reefs. Academic Press, San Diego, pp. 475-508.

Jacques, G., Treguer, P., 1986. Ecosystèmes pélagiques marins. Masson (Ed.), pp. 243.

Jones, G.P., Milicich, M.J., Emslie, M.J., Lunow, C., 1999. Selfrecruitment in a coral reef fish population. Nature 402, 802-805.

Lasker, R., 1989. Les déterminants du recrutement. In : Troadec, J.P (Ed.), L'homme et les ressources halieutiques. Bull. Ifremer. pp. 329-374.

Letourneur, Y., 1992. Dynamique des peuplements ichtyologiques des platiers récifaux de l'île de La Réunion. Thèse Océanogr. biol., Univ. Aix-Marseille II. pp. 244.

Letourneur, Y., 1996. Dynamics of fish communities on Reunion fringing reefs, Indian Ocean. Patterns of spatial distribution. J. Exp. Mar. Biol. Ecol. 195, 1-30.

Letourneur, Y., Chabanet, P., Vigliola, L., Harmelin-Vivien, M., 1998. Mass settlement and post-settlement mortality of Epinephelus merra (pisces: Serranidae) on Reunion coral reefs. J. Mar. Biol. Ass. 78, 307-319.

Luckurst, B.E., Luckurst, K., 1977. Recruitment patterns of Coral Reef Fishes of the fringing reef of Curacao Netherland Antilles. Can. J. Zool. 55, 681-689.

Mc Farland, W.N., Ogden, J.C., 1985. Recruitment of young coral reef fishes from the plankton. In : Reaka, M.L (Ed.), The ecology of coral reefs. NOAA Symp. Ser., 3, pp. 37-51.

Mc Ilwain, J.L., 1997. Hydrodynamic flows and the flux of larval fishes across the crest of Ningaloo Reef, Western Australia. Proc. $8^{\text {th }}$ Int. Coral Reef Symp. 2, 1133-1138.

Planes, S., Galzin, R., Bonhomme, F., 1996. A genetic metapopulation model for reef fishes in oceanic islands: the case of the surgeonfish Acanthurus triostegus. J. Evol. Biol. 9, 103-117.

Russel, B.C., Talbot, F.H., Anderson, G.R.V., 1977. Seasonality and recruitment of coral reef fishes. Aust. J. Mar. Fresh. Res. 28, 521-528.

Sale, P.F., 1980. The ecology of fishes on coral reefs. Oceanogr. Mar. Biol. $18,367-421$.

Sale, P.F., Doherty, P.J., Eckert, G.J., Douglas, W.A., Ferrel, D.J., 1984. Large scale spatial and temporal variation in recruitment to fish populations on coral reefs. Oecologia 64, 191-198.

Sale, P.F., Douglas, W.A., 1984. Temporal variability in the community structure of fishes on coral reefs, and the relation of community structure to reef structure. Ecology 65, 409-422.

Shima, J., 1999. Variability in relative importance of determinants of reef fish recruitment. Ecol. lett. 2, 304-310.

Shulman, M.J., Ogden, J.C., Ebersole, J.P., Mc Farland, W.N., Miller, S.L., Wolf, N.G., 1984. Priority effects in the recruitment of juvenile coral reef fishes. Ecology 64, 1508-1513.

Swearer, S.E., Caselle, J.E., Lea, D.W., Warner, R.R., 1999. Larval retention and recruitment in an island population of a coral reef fish. Nature 402, 799-802.

Victor, B.C., 1986. Larval settlement and juvenile mortality in a recruitment-limited coral reef fishes population. Ecol. Monogr. 56, $145-160$.

Walsh, W.J., 1987. Patterns of recruitment and spawning in Hawaiian reef fishes. Environ. Biol. Fish. 18, 257-276.

Williams, D.McB., 1983. Daily, monthly and yearly variability in recruitment of a guild of coral reef fishes. Mar. Ecol. Prog. Ser. 10, 231-237. 